\title{
Random iteration of analytic maps
}

\author{
A. F. BEARDON $\dagger$, T. K. CARNE $\dagger$, D. MINDA $\neq$ and T. W. NG $\S$ \\ $\dagger$ Centre for Mathematical Studies, University of Cambridge, Wilberforce Road, \\ Cambridge CB3 OWB, UK \\ (e-mail:afb@dpmms.cam.ac.uk,tkc@dpmms.cam.ac.uk) \\ $\ddagger$ Mathematical Sciences Department, University of Cincinnati, PO Box 210025, \\ Cincinnati, OH 45221-0025, USA \\ (e-mail:david.minda@math.uc.edu) \\ $\S$ Department of Mathematics, The University of Hong Kong, Pokfulam Road, Hong Kong \\ (e-mail:ntw@maths.hku.hk)
}

(Received 2 August 2000 and accepted in final form 15 January 2004)

\begin{abstract}
We consider analytic maps $f_{j}: D \rightarrow D$ of a domain $D$ into itself and ask when does the sequence $f_{1} \circ \cdots \circ f_{n}$ converge locally uniformly on $D$ to a constant. In the case of one complex variable, we are able to show that this is so if there is a sequence $\left\{w_{1}, w_{2}, \ldots\right\}$ in $D$ whose values are not taken by any $f_{j}$ in $D$, and which is homogeneous in the sense that it comes within a fixed hyperbolic distance of any point of $D$. The situation for several complex variables is also discussed.
\end{abstract}

\section{Introduction}

Given a mapping $f: X \rightarrow X$ one can study the iterates $f^{n}: X \rightarrow X$ of $f$. More generally, if $\mathcal{F}$ is a family of maps of $X$ into itself, one can study the behaviour of the sequences $f_{1} \circ \cdots \circ f_{n}$ as $n \rightarrow \infty$, where the $f_{j}$ are chosen from $\mathcal{F}$. This process is often referred to as an iterated function system or, if the $f_{j}$ are chosen with certain probabilities, as random iteration. The sequences $f_{1} \circ \cdots \circ f_{n}$ arise naturally in dynamical systems, in continued fraction theory (where the $f_{n}$ are Möbius transformations), and in certain questions in complex analysis. We are concerned here with finding conditions that imply that if $f_{1}, f_{2}, \ldots$ are maps of a metric space $X$ into itself, then every sequence $f_{1} \circ \cdots \circ f_{n}$ converges locally uniformly on $X$ to some constant function. Although we shall briefly consider the general problem, our main concern is the application of these ideas to analytic functions of one or more complex variables.

We begin with a modest generalization of the well-known contraction mapping theorem in a form that is applicable to random iteration and that includes the contraction mapping theorem as a special case (when $f_{j}=f$ for all $j$ ). 
THEOREM 1.1. Suppose that $(X, d)$ is a complete metric space, and that $0<k<1$, and let $f_{1}, f_{2}, \ldots$ be a sequence of maps of $X$ into itself each of which satisfies the uniform Lipschitz condition $d\left(f_{j}(x), f_{j}(y)\right) \leq k d(x, y)$ for all $x$ and $y$ in $X$. Suppose also that there exists some $x_{0}$ in $X$ such that the sequence $f_{1}\left(x_{0}\right), f_{2}\left(x_{0}\right), \ldots$ is bounded. Then the sequence $f_{1} \circ \cdots \circ f_{n}$ converges locally uniformly on $X$ to a constant function.

The proof of this follows shortly. Our aim is to find criteria that will enable us to apply Theorem 1.1 and so obtain the convergence of each sequence $f_{1} \circ \cdots \circ f_{n}$ to a constant. As many complex analytic maps are contractions (with respect to hyperbolic metrics) it is natural to focus on analyticity, and we begin by considering analytic functions of one complex variable. Later we consider functions of several complex variables. First, we recall the well known Denjoy-Wolff Theorem on the iteration of analytic maps; in this the iterates converge to a constant limit without any assumption of a Lipschitz condition.

The DenJoY-WolfF Theorem. Let $\mathbb{D}$ be the open unit disc in the complex plane $\mathbb{C}$, and let $f$ be any analytic map of $\mathbb{D}$ into itself that is not a conformal automorphism of $\mathbb{D}$. Then the iterates $f^{n}$ of $f$ converge locally uniformly in $\mathbb{D}$ to a constant value $\zeta$, where $|\zeta| \leq 1$.

A few remarks may be helpful. First, whereas Theorem 1.1 will necessarily produce constant limit functions whose value lies in $X$, the Denjoy-Wolff Theorem allows the constant values to lie on the boundary of $\mathbb{D}$, and it is here that the hyperbolic nature of $\mathbb{D}$ is crucial. Briefly, the disc $\mathbb{D}$ is equipped with the hyperbolic metric $2|d z| /\left(1-|z|^{2}\right)$, and the resulting metric space with metric $\rho_{\mathbb{D}}$, where

$$
\rho_{\mathbb{D}}(z, w)=2 \tanh ^{-1}\left|\frac{z-w}{1-\bar{w} z}\right|,
$$

is complete. An informal explanation of the Denjoy-Wolff Theorem is that the negative curvature of $\rho_{\mathbb{D}}$ means that the space $\mathbb{D}$ is 'expanding' rapidly near the circle at infinity, and the Schwarz-Pick lemma implies that any analytic map $f: \mathbb{D} \rightarrow \mathbb{D}$ is contracting in the sense that for all $z$ and $w$ in $\mathbb{D}$,

$$
\rho_{\mathbb{D}}(f(z), f(w))<\rho_{\mathbb{D}}(z, w)
$$

(unless $f$ is an isometry, and hence a Möbius map of $\mathbb{D}$ onto itself). Together these facts force the convergence (in the Euclidean metric) of $f^{n}$ to a constant. In fact, this result has nothing to do with analyticity for it is equally valid for any map $f: \mathbb{D} \rightarrow \mathbb{D}$ that is contracting with respect to $\rho_{\mathbb{D}}$. For more information on the Denjoy-Wolff Theorem, see $[1,7,10]$ and references therein. For other results that are indirectly related to the general problem considered here see $[\mathbf{6 - 1 0 , 1 9 ]}$. Generalizations of the Denjoy-Wolff Theorem to other spaces are contained in $[\mathbf{7}, \mathbf{1 0}]$, and $[\mathbf{6}, \mathbf{8}, \mathbf{9}]$ discuss the iterates of an analytic map $f$ of a domain $D$ into itself that is a Euclidean contraction; that is, a map $f$ that satisfies $|f(z)-f(w)|<|z-w|$ in $D$.

Let us now turn to questions concerning the random iteration of analytic functions of one variable. In recent years several papers have appeared on this question (see, for example, $[2,3,11]$, which are concerned with ergodic questions, and also $[5,15,16,18]$ and [19], which gives a survey of such results in the context of continued fractions). These latter 
papers are closer to the work in this paper, but only apply to the case in which the $f_{j}$ are analytic maps of a plane domain $D$ into a compact subset $K$ of $D$ (this is also used in [2, p. 1387]), and $D$ is (essentially) assumed to be simply connected. To be specific, we have the following result [18, Theorem 1] which generalizes an earlier result due to Gill [15].

Theorem A. Suppose that $D$ and $D_{0}$ are domains in $\mathbb{C}$, and that $K \subset D_{0} \subset D \subset \mathbb{C}$, where $D_{0}$ is simply connected, and $K$ is a compact subset of $D_{0}$. Suppose also that $f_{1}, f_{2}, \ldots$ are analytic maps of $D$ into $K$. Then $f_{1} \circ \cdots \circ f_{n}$ converges to a constant locally uniformly in $D$.

Although $D$ may be multiply connected here, the assumption that such a simply connected $D_{0}$ exists prevents the application of this result to a compact subset $K$ of $D$ that separates the complement of $D$. Moreover, if we know that the corresponding result holds for any simply connected domain, the assumptions imply that the sequence $f_{1} \circ \cdots \circ f_{n}$ converges locally uniformly on $D_{0}$ to a constant function, and it now follows (from the theory of normal families, provided only that $\mathbb{C} \backslash D$ contains at least two points) that $f_{1} \circ \cdots \circ f_{n}$ converges locally uniformly on $D$ to a constant function. We remark that there is a similar result for several variables in [25] (Theorem $\mathrm{C}$ in this paper) which, when specialized to one variable, applies to bounded (possibly multiply connected) domains but still with the $f_{j}$ mapping into a compact subset of $D$.

Theorem A also occurs, with the assumption that $D$ is simply connected (and hence no mention of $D_{0}$ ), as Corollary 2.3 in [5], where it is derived from a result of a quite different nature concerning the possible limits of sequences of the form $f_{n_{j}} \circ \cdots \circ f_{n_{k}}$. On p. 186 of [5], Baker and Rippon comment that the hypothesis that the $f_{j}$ map $D$ into a compact set $K$ 'seems stringent when compared with the original Denjoy-Wolff Theorem', and that 'It is tempting to hope that some weaker condition on $K$ will yield the conclusion of the corollary'. They then state that 'the illusory nature of this hope ... is shown by the following example' [5, Example 2.6, p. 186]. Our results here show that considerable progress can be made in weakening the assumption that $K$ is compact.

We turn now to the main results in this paper. First, we recall that a subdomain $D$ of $\mathbb{C}$ is hyperbolic if it supports a complete Riemannian metric of constant curvature -1 , namely the (unique) hyperbolic metric $\lambda_{D}(z)|d z|$, and it is well known that this is so if and only if its complement in $\mathbb{C}$ contains at least two points. We use $\rho_{D}$ for the induced hyperbolic distance in $D$. Now the general form of the Schwarz-Pick lemma states that any analytic map $f: D_{1} \rightarrow D_{2}$ between two hyperbolic domains $D_{1}$ and $D_{2}$ is contracting in the weak sense that $\rho_{2}(f(z), f(w)) \leq \rho_{1}(z, w)$, where $\rho_{j}$ is the hyperbolic metric on $D_{j}$. It is not difficult to see that if $f$ maps $D$ into a compact subset $K$ of $D$, then $f$ satisfies the stronger contractive property that is required in the contraction mapping theorem and, as the scaling factor $k$ depends only on $D$ and $K$, this enables us to apply Theorem 1.1 and so derive results on random iteration. The essential ideas in this paper are: (1) that these arguments remain valid for maps $f$ of $D$ into an arbitrary subset $E$ of $D$ provided only that the geometry of $E$ guarantees this strong contractive property of $f$; and (2) that there is a simple geometric condition on $E$ that guarantees this strong contractive property, and which is satisfied by many more subsets of $D$ than just the compact subsets of $D$. 
To the best of our knowledge, this is the first time that non-compact subsets of $D$ have been considered in this context. The introduction of the hyperbolic metric is crucial here because we want to use Theorem 1.1 and one of the major deficiencies of the Euclidean metric is that its restriction to a proper subdomain of $\mathbb{C}$ is not complete.

Suppose that $\Omega$ is a subdomain of a hyperbolic domain $D$. Then $\Omega$ is also hyperbolic, and the Schwarz-Pick lemma applied to the identity (inclusion) map yields the monotonicity principle for the hyperbolic metric, namely that $\lambda_{D} \leq \lambda_{\Omega}$ on $\Omega$ with a strict inequality at each point of $\Omega$ when $\Omega \neq D$. It is convenient to write

$$
\mu(\Omega, D)=\sup _{w \in \Omega} \frac{\lambda_{D}(w)}{\lambda_{\Omega}(w)}
$$

thus $\mu(\Omega, D) \leq 1$. Of course, it may be that $\lambda_{D}(z)<\lambda_{\Omega}(z)$ for each $z$ in $\Omega$, yet $\mu(\Omega, D)=1$, and this suggests the next definition.

Definition 1.2. The subdomain $\Omega$ is a Lipschitz subdomain of $D$ if $\mu(\Omega, D)<1$.

We shall see later that this leads easily to the next result.

THEOREM 1.3. Suppose that $f_{1}, f_{2}, \ldots$ are analytic maps of a hyperbolic plane domain $D$ into a Lipschitz subdomain $\Omega$ of $D$. Then every limit function of the sequence $f_{1} \circ \cdots \circ f_{n}$ is constant. Suppose that, in addition, for some $z_{0}$ in $D$ the sequence $f_{n}\left(z_{0}\right)$ lies in a compact subset of $D$; then $f_{1} \circ \cdots \circ f_{n}$ converges locally uniformly on $D$ to a constant function.

It is easy to see that if $\Omega$ lies in a compact subset of $D$ then $\Omega$ is a Lipschitz domain, and later we shall give many examples of Lipschitz subdomains that are not relatively compact in $D$. Thus Theorem 1.3 is a very significant strengthening of the earlier results of this type. It gives a very general condition under which all limit functions are constant, and here the domain $D$ may be multiply connected, and the $f_{j}(D)$ may lie in a 'large' (i.e. non-compact) subset of $D$. In fact, the proof of Theorem 1.3 will show that a similar result holds in the more general circumstances in which each $f_{j}$ is an analytic map of $D$ into $\Omega_{j}$ providing only that there is some constant $k$ such that for all $j, \mu\left(\Omega_{j}, D\right) \leq k<1$. Theorem 1.3 is the case when $\Omega_{j}=\Omega$ for all $j$.

In order to be able to apply Theorem 1.3 , we need a criterion that enables us to recognize when a subdomain of $D$ is a Lipschitz subdomain, and before we can state our criterion we need to introduce the idea of a Bloch domain. We recall that a subdomain $\Omega$ of $\mathbb{C}$ is a Bloch domain if and only if there is a finite upper bound on the radii of the Euclidean discs that lie in $\Omega$. We now introduce the analogous definition for the hyperbolic metric.

Definition 1.4. A subdomain $\Omega$ of a hyperbolic domain $D$ in $\mathbb{C}$ is a Bloch subdomain of $D$ if and only if there is a finite upper bound on the radii of the discs (in the metric $\rho_{D}$ ) lying in $\Omega$.

Suppose now that $\Omega$ is a subdomain of $D$, and consider the collection $\mathcal{D}$ of open discs in the metric space $\left(D, \rho_{D}\right)$. We denote the radius (as measured by $\rho_{D}$ ) of the open disc $\Delta$ in $\mathcal{D}$ by $r_{\Delta}$. Now let

$$
R(\Omega, D)=\sup \left\{r_{\Delta}: \Delta \in \mathcal{D}, \Delta \subset \Omega\right\},
$$


where, of course, we allow $R(\Omega, D)$ to be $+\infty$. Clearly, $\Omega$ is a Bloch subdomain of $D$ if and only if $R(\Omega, D)<+\infty$. The following theorem shows (quantitatively) that a subdomain of $D$ is a Lipschitz subdomain if and only if it is a Bloch subdomain.

THEOREM 1.5. Suppose that $\Omega$ is a subdomain of a hyperbolic plane domain $D$; then

$$
\tanh \frac{1}{2} R(\Omega, D) \leq \mu(\Omega, D) \leq \tanh R(\Omega, D)
$$

In particular, $\Omega$ is a Lipschitz subdomain of $D$ if and only if $R(\Omega, D)<+\infty$.

We can now rewrite Theorem 1.3 in the following form, and this is the main result in this paper.

THEOREM 1.6. Suppose that $\Omega$ is a Bloch subdomain of a hyperbolic domain $D$ and that $f_{1}, f_{2}, \ldots$ are analytic maps of $D$ into $\Omega$. Then any limit function of $f_{1} \circ \cdots \circ f_{n}$ is constant. Further, if there is some $z_{0}$ in D such that the sequence $f_{n}\left(z_{0}\right)$ lies in a compact subset of $D$, then $f_{1} \circ \cdots \circ f_{n}$ converges locally uniformly on $D$ to a constant function.

As a special case of Theorem 1.6, we have the following result.

THEOREM 1.7. Suppose that $D$ is a hyperbolic subdomain of $\mathbb{C}$, and let $z_{n}$ be a sequence in $D$ that accumulates only on $\partial D$, and meets every hyperbolic disc in $D$ of some given radius $d$, say. Let $\Omega=D \backslash\left\{z_{1}, z_{2}, \ldots\right\}$. Then for any collection of analytic maps $f_{j}$ of $D$ into $\Omega$, any limit function of $f_{1} \circ \cdots \circ f_{n}$ is constant. Further, if there is some $z_{0}$ in $D$ such that the sequence $f_{n}\left(z_{0}\right)$ lies in a compact subset of $D$, then $f_{1} \circ \cdots \circ f_{n}$ converges locally uniformly on $D$ to a constant function.

There are several straightforward but interesting consequences of Theorem 1.6, and in describing these we assume a modest familiarity with hyperbolic geometry. Of course, the most striking example is that described in Theorem 1.7. The existence of such a sequence $z_{n}$ in Theorem 1.7 (indeed, of many such sequences) is easily established. We choose any point $a$ in $D$, and let $U_{n}$ and $K_{n}$ be the open disc and closed disc, respectively, with centre $a$ and radius $n$ (measured with respect to $\rho_{D}$ ). Now cover the compact set $K_{1}$ by a finite number of discs of radius one. Next, cover the compact set $K_{2} \backslash U_{1}$ by a finite number of discs of radius one, then $K_{3} \backslash U_{2}$ and so on. The set of centres of all of these discs, for all $n$, may now be taken as the sequence $z_{j}$ as this sequence clearly accumulates only on $\partial D$ and $D \backslash\left\{z_{1}, z_{2}, \ldots\right\}$ is a Bloch domain. A similar construction shows that for each compact subset $K$ there is a positive $d$ and a sequence $z_{n}$ of points in $D \backslash K$, that accumulates only on $\partial D$, and that meets every hyperbolic disc in $D$ with radius $d$.

We briefly mention some other examples of Lipschitz subdomains of the unit disc $\mathbb{D}$. A horodisc (an open Euclidean disc that is internally tangent to the unit circle $\partial \mathbb{D}$ ) is not a Lipschitz subdomain of $\mathbb{D}$ as it contains arbitrarily large $\rho_{\mathbb{D}}$-discs. In contrast, the region lying between two circles that are tangent to $\partial \mathbb{D}$ at the same point is a Lipschitz subdomain of $\mathbb{D}$. Next, a hyperbolic Stolz region (the region formed by taking the union of all hyperbolic discs of a fixed radius whose centres lie on a hyperbolic geodesic in $\mathbb{D}$ ) is a Lipschitz domain. Finally, a hyperbolic polygon in $\mathbb{D}$ (the hyperbolic convex hull of a finite set of points in the closed unit disc) is also a Lipschitz subdomain of $\mathbb{D}$. 
We now turn to discuss the results for several complex variables. Throughout this discussion $X$ and $Y$ are complex Banach spaces and $\mathcal{H}(X, Y)$ denotes the space of holomorphic (i.e. analytic) maps of $X$ into $Y$. We follow the customary terminology (in this area) by calling a metric (in the sense of metric spaces) a distance, and by using metric for the infinitesimal version of this. A pseudo-distance $d$ satisfies the usual requirements of a distance except that $d(x, y)=0$ does not imply that $x=y$. For more details about this section, we refer the reader to $[\mathbf{1 2}, \mathbf{1 4}, \mathbf{1 7}, \mathbf{2 4}$ ) (and in particular to chapters IV and V in [14]). We remark that most of these ideas can be discussed for holomorphic maps between complex manifolds (including Riemann surfaces), but as we have no new ideas to introduce in this context we confine our discussion to Banach spaces.

We begin with the following fixed point theorem of Earle and Hamilton, which is another modification of the contraction mapping theorem (see [13] and [14, pp. 91, 137-139]).

THEOREM B. Let $K$ be a compact subset of a bounded domain $D$ in a complex Banach space, and suppose that $f: D \rightarrow D$ is holomorphic and that $f(D) \subset K$. Then $f$ has a unique fixed point, say $\zeta$, in $D$, and $f^{n} \rightarrow \zeta$ in $D$.

This result has been extended by Zhang and Ren [25] to a result that applies to random iteration.

THEOREM C. Let $D$ be a bounded domain in a complex Banach space $X$, and suppose that $K$ is a compact subset of D. Suppose also that $f_{1}, f_{2}, \ldots$ are holomorphic self-maps of $D$, and that for each $j, f_{j}(D) \subset K$. Then $f_{1} \circ \cdots \circ f_{n}$ converges uniformly on $D$ to a constant.

The proof of Theorem C follows closely from the proof of Theorem B that is given in [14], but see also [25] (in particular Remark 2.1 on p. 35). The crucial role of the hyperbolic metric in one complex dimension is taken over in the proof of Theorem $\mathrm{C}$ by the Kobayashi metric, and we now give a brief description of this. For each $x$ and $y$ in a domain $D$ in a complex Banach space $X$, let

$$
\delta_{D}(x, y)=\inf \left\{\rho_{\mathbb{D}}(u, v): f \in \mathcal{H}(\mathbb{D}, D), f(u)=x, f(v)=y\right\}
$$

The set on the right-hand side is non-empty (so that $\delta_{D}$ is defined), and it is clear that $\delta_{D}$ is symmetric. However, $\delta_{D}$ need not satisfy the triangle inequality, and to overcome this Kobayashi introduced the Kobayashi pseudo-distance $k_{D}$ by

$$
k_{D}(u, v)=\inf \sum_{j=1}^{n} \delta_{D}\left(w_{j}, w_{j+1}\right),
$$

where this infimum is taken over all finite sequences $w_{1}, \ldots, w_{n+1}$ (for any $n$ ) in $D$ such that $w_{1}=u$ and $w_{n+1}=v$. It is known that $k_{D}$ is a pseudo-distance on $D$. The essential role of the Schwarz-Pick lemma remains true in these circumstances, that is holomorphic maps are (weak) contractions with respect to the Kobayashi pseudo-distances; explicitly, if $f \in \mathcal{H}\left(D, D^{\prime}\right)$, then

$$
k_{D^{\prime}}(f(x), f(y)) \leq k_{D}(x, y)
$$


By taking the holomorphic map to be the identity, the monotonicity property of the pseudodistance follows; that is, if $D_{1} \subset D$, then $k_{D} \leq k_{D_{1}}$.

Next, we consider the infinitesimal Kobayashi metric on a domain $D$ in a Banach space $X$; this is the generalization of the infinitesimal hyperbolic metric $\lambda_{D}(z)|d z|$. The Kobayashi seminorm $K_{D}$ is defined at each point $(p, v)$ of the tangent bundle $T(X)$ by

$$
K_{D}(p, v)=\inf \{x>0 \text { : there exists } f \text { in } \mathcal{H}(\mathbb{D}, D), f(0)=p, d f(0)(x)=v\},
$$

and Royden [24] proved that

$$
k_{D}(p, q)=\inf _{\gamma} \int_{0}^{1} K_{D}\left(\gamma(t), \gamma^{\prime}(t)\right) d t
$$

where the infimum is taken over all smooth paths $\gamma:[0,1] \rightarrow X$ joining $p$ to $q$. We say that the domain $D$ is Kobayashi hyperbolic if $k_{D}$ is a distance (that is, if $k_{D}(x, y)>0$ when $x \neq y)$, and in this case $K_{D}(p, v)>0$ (see [17, Theorem 2, p. 133]). We remark that the Kobayashi metric on even a bounded subdomain of $\mathbb{C}^{N}$ may not be complete, and this fact highlights one of the major differences between the theory of one and several variables.

Clearly, $K_{D}$ is contracting with respect to holomorphic maps; explicitly, if $f \in$ $\mathcal{H}\left(D, D^{\prime}\right)$, then for all $x$ in $D$ and all $v$ in $X, K_{D^{\prime}}(f(x), d f(x)(v)) \leq K_{D}(x, v)$. As before, this implies that if $D \subset D^{\prime}$, then $K_{D^{\prime}}(x, v) \leq K_{D}(x, v)$. In view of our earlier discussion, it is now natural to make the following definition.

Definition 1.8. A subdomain $\Omega$ of a domain $D$ in a Banach space $X$ is a Lipschitz subdomain of $D$ if $D$ is Kobayashi hyperbolic, and if

$$
\mu(\Omega, D)=\sup \left\{\frac{K_{D}(p, v)}{K_{\Omega}(p, v)}:(p, v) \in T(\Omega), v \neq 0\right\}<1
$$

The next result follows easily from this.

THEOREM 1.9. Suppose that $X$ is a complex Banach space, and that $f_{1}, f_{2}, \ldots$ are holomorphic maps from a bounded, Kobayashi hyperbolic subdomain $D$ of $X$ into a Lipschitz subdomain $\Omega$ of $D$. Then $f_{1} \circ \cdots \circ f_{n}$ converges locally uniformly on $D$ to a constant.

In this result the constant limit may lie on the boundary of $D$, and if we take $f_{1}=f_{2}=$ $\cdots=f$ here, we obtain a type of Denjoy-Wolff Theorem for bounded subdomains of complex Banach spaces.

It is easy to see that if $\Omega$ is a relatively compact subdomain of a bounded domain $D$ in a complex Banach space, then $\Omega$ is a Lipschitz subdomain of $D$ (this is essentially the proof of Theorem C); thus (in a rather trivial sense) Theorem 1.9 contains Theorem $\mathrm{C}$.

Of course, these results raise the obvious question, namely is there a characterization of Lipschitz subdomains in a Banach space that corresponds to the notion of a Bloch domain described earlier? We show that the answer here is 'no' as we give an example of a bounded subdomain of $\mathbb{C}^{2}$ that is (in the obvious sense) a Bloch domain but not a Lipschitz subdomain. In short, Theorem 1.5 does not generalize to more than one variable and as 
this lies at the heart of our geometric recognition of Lipschitz domains in $\mathbb{C}$, there are still open questions about what these look like in $\mathbb{C}^{N}$, where $N \geq 2$.

We give the proof of Theorem 1.1 in $\S 2$. In $\S 3$ we study the random iteration of a family of analytic maps which is assumed to satisfy a uniform Lipschitz condition and we prove Theorem 1.3. The proof of Theorem 1.5 is given in $\S \S 4$ and 5. We prove Theorem 1.9 in $\S 6$ and, finally, in $\S 7$ we give an example of a Bloch subdomain of $\mathbb{D} \times \mathbb{D}$ that is not a Lipschitz subdomain.

\section{The proof of Theorem 1.1}

This section is devoted to the proof of Theorem 1.1. As the sequence $f_{n}\left(x_{0}\right)$ is bounded, the sequence $d\left(x_{0}, f_{j}\left(x_{0}\right)\right), j=1,2, \ldots$, is also bounded, say by $A$. Now for any $x$ in $X$,

$$
d\left(x, f_{j}(x)\right) \leq d\left(x, x_{0}\right)+d\left(x_{0}, f_{j}\left(x_{0}\right)\right)+d\left(f_{j}\left(x_{0}\right), f_{j}(x)\right)<2 d\left(x_{0}, x\right)+A,
$$

so that for each $x$, the sequence $d\left(x, f_{j}(x)\right)$ is bounded. Take any $x$ in $X$ and, for brevity, write $y_{n}=f_{1} \circ \cdots \circ f_{n}(x)$. Then, for any natural numbers $m$ and $n$,

$$
d\left(y_{n}, y_{n+m}\right) \leq \sum_{j=n}^{n+m-1} d\left(y_{j}, y_{j+1}\right) \leq \sum_{j=n}^{n+m-1} k^{j} d\left(x, f_{j+1}(x)\right) \leq \frac{k^{n}}{1-k}\left(2 d\left(x, x_{0}\right)+A\right) .
$$

As $(X, d)$ is complete, this shows that the sequence $y_{n}$ converges, and hence that the sequence $f_{1} \circ \cdots \circ f_{n}$ converges pointwise on $X$, say to the function $g$. As

$$
d\left(f_{1} \circ \cdots \circ f_{n}(x), f_{1} \circ \cdots \circ f_{n}(y)\right) \leq k^{n} d(x, y) \rightarrow 0
$$

as $n \rightarrow \infty$, we see that $g$ is constant on $X$, with a value of $\zeta$, say. Now take any $a$ in $X$ and any compact subset $K$ of $X$, and let $d_{a}=\sup _{x \in K} d(a, x)$ (which is finite). Then for all $x$ in $K$ we have

$$
d\left(f_{1} \circ \cdots \circ f_{n}(a), f_{1} \circ \cdots \circ f_{n}(x)\right) \leq k^{n} d(a, x) \leq k^{n} d_{a},
$$

so that $f_{1} \circ \cdots \circ f_{n} \rightarrow \zeta$ locally uniformly on $X$. The proof is complete.

Of course, the extent to which Theorem 1.1 is useful depends on our ability to find (preferably geometric) conditions under which a family of maps from $X$ to itself is uniformly Lipschitz. The rest of the paper is concerned with this problem.

Remark. Given any sequence $f_{1}, f_{2}, \ldots$ of self-maps of $(X, d)$, we say that $g$ is a limit function of the sequence $f_{n}$ if there is a subsequence of this sequence that converges locally uniformly on $X$ to $g$. This paper is about the possible limit functions of the sequence $f_{1} \circ \cdots \circ f_{n}$. We note that the last part of the proof of Theorem 1.1 shows that even when $(X, d)$ is not complete, if the sequence $f_{1} \circ \cdots \circ f_{n}$ converges pointwise on $X$, then it converges locally uniformly on $X$ to a constant. Thus if $(X, d)$ is any metric space and if the family $\left\{f_{j}\right\}$ of self-maps of $X$ is uniformly Lipschitz in the sense of Theorem 1.1, then the sequence $f_{1} \circ \cdots \circ f_{n}$ can only have constant limit functions. Situations such as these are common in the study of dynamical systems; for example, if $X=[0,1]$ with the Euclidean metric, and $\mathcal{F}=\left\{f_{1}, f_{2}\right\}$, where $f_{1}(x)=x / 3$ and $f_{2}(x)=(2+x) / 3$, then the possible constant limit functions are those whose value lies in the classical Cantor set. 
3. Random iteration of uniformly Lipschitz families of analytic maps

In this section we explain our terminology in Definition 1.2 and prove Theorem 1.3.

We begin by noting that $\mu(\Omega, D)$ is conformally invariant (and this is another reason for using the hyperbolic rather than the Euclidean metric). Indeed, if $f$ is any conformal map of $D$ onto the domain $f(D)$ (so that $f$ is also a conformal map of $\Omega$ onto $f(\Omega)$ ) then, for every $z$ in $\Omega$,

$$
\begin{aligned}
& \lambda_{f(D)}(f(z))\left|f^{\prime}(z)\right|=\lambda_{D}(z), \\
& \lambda_{f(\Omega)}(f(z))\left|f^{\prime}(z)\right|=\lambda_{\Omega}(z),
\end{aligned}
$$

and hence

$$
\mu(f(\Omega), f(D))=\mu(\Omega, D)
$$

(we generalize this result to covering maps later). It follows that the relationship of $\Omega$ being a Lipschitz subdomain of $D$ is also conformally invariant. In addition, it is immediate from the definition of a Lipschitz subdomain and the monotonicity principle that any subdomain of a Lipschitz subdomain is also Lipschitz; indeed, if $\Omega^{\prime} \subset \Omega \subset D$, then $\mu\left(\Omega^{\prime}, D\right) \leq \mu(\Omega, D)$. The following lemma allows us to apply Theorem 1.1.

LEMMA 3.1. Suppose that $\Omega$ is a Lipschitz subdomain of $D$. Then the family of analytic maps from $\left(D, \rho_{D}\right)$ to $\left(\Omega, \rho_{D}\right)$ is uniformly Lipschitz with Lipschitz constant $\mu(\Omega, D)$; that is, for each analytic $f: D \rightarrow \Omega$ and each $z$ and $w$ in $D, \rho_{D}(f(z), f(w)) \leq$ $\mu(\Omega, D) \rho_{D}(z, w)$.

Proof. Suppose that $\Omega$ is a Lipschitz subdomain of $D$, and that $f: D \rightarrow \Omega$ is any analytic map. Let $z$ and $w$ be any two points in $D$, and let $\gamma$ be the geodesic in the metric $\rho_{\Omega}$ that joins $f(z)$ to $f(w)$ in $\Omega$. Then

$$
\begin{aligned}
\rho_{D}(f(z), f(w)) & \leq \int_{\gamma} \lambda_{D}(\zeta)|d \zeta| \\
& \leq \mu(\Omega, D) \int_{\gamma} \lambda_{\Omega}(\zeta)|d \zeta| \\
& =\mu(\Omega, D) \rho_{\Omega}(f(z), f(w)) \\
& \leq \mu(\Omega, D) \rho_{D}(z, w)
\end{aligned}
$$

as required, the last inequality following directly from the general form of the SchwarzPick lemma. The proof of Lemma 3.1 is complete.

If we combine Theorem 1.1 and the Remark at the end of $\$ 2$ with Lemma 3.1, we immediately obtain Theorem 1.3.

\section{Universal cover maps}

Throughout this short section we suppose that $D$ is a hyperbolic domain in $\mathbb{C}$, and that $\Omega$ is a proper subdomain of $D$. We take any point $a$ in $\Omega$, let $\pi: \mathbb{D} \rightarrow D$ be a universal covering projection with $\pi(0)=a$, and let $\Sigma$ be the component of $\pi^{-1}(\Omega)$ that contains the origin. The next result generalizes (3.1) and, in effect, implies that when proving Theorem 1.5 we may assume that $D=\mathbb{D}$. 
LEMMA 4.1. In the notation above, $\mu(\Omega, D)=\mu(\Sigma, \mathbb{D})$.

Proof. As $\pi: \mathbb{D} \rightarrow D$ is a universal covering projection, $\lambda_{D}(\pi(z))\left|\pi^{\prime}(z)\right|=\lambda_{\mathbb{D}}(z)$, and as $\pi \mid \Sigma$ is a covering projection of $\Sigma$ onto $\Omega$, we have $\lambda_{\Omega}(\pi(z))\left|\pi^{\prime}(z)\right|=\lambda_{\Sigma}(z)$. Thus

$$
\frac{\lambda_{D}(\pi(z))}{\lambda_{\Omega}(\pi(z))}=\frac{\lambda_{\mathbb{D}}(z)}{\lambda_{\Sigma}(z)},
$$

and because $\pi$ maps $\Sigma$ onto $\Omega$, this implies that $\mu(\Omega, D)=\mu(\Sigma, \mathbb{D})$ as required.

Next, given any pair $\Omega$ and $D$, and $z$ in $\Omega$, let $R(z ; \Omega, D)$ be the radius of the largest open disc (relative to the hyperbolic metric on $D$ ) with centre $z$ that is contained in $\Omega$; thus (see (1.1))

$$
R(\Omega, D)=\sup \{R(z ; \Omega, \mathbb{D}): z \in \Omega\} .
$$

We now show that this too is invariant under a cover map.

Lemma 4.2. In the notation above, $R(\Omega, D)=R(\Sigma, \mathbb{D})$.

Proof. It is convenient to use the notation $\Delta_{D}(z, r)$ to denote the open disc (relative to the hyperbolic metric on $D$ ) with centre $z$ and radius $r$. As any point of $\Delta_{\mathbb{D}}(z, r)$ can be joined to $z$ by an arc of length less than $r$, and as $\pi$ is a local isometry, it is immediate that

$$
\pi\left(\Delta_{\mathbb{D}}(z, r)\right) \subset \Delta_{D}(\pi(z), r) .
$$

A similar argument holds for $\pi^{-1}$ (as all curves lift under $\pi^{-1}$ ), so we find that

$$
\pi\left(\Delta_{\mathbb{D}}(z, r)\right)=\Delta_{D}(\pi(z), r) .
$$

Suppose now that $\Delta_{\mathbb{D}}(z, r) \subset \Sigma$. Then, from (4.2), $\Delta_{D}(\pi(z), r) \subset \Omega$, and this implies that

$$
R(z ; \Sigma, \mathbb{D}) \leq R(\pi(z) ; \Omega, D) .
$$

On the other hand, if $\Delta_{D}(\pi(z), r) \subset \Omega$, then $\pi\left(\Delta_{\mathbb{D}}(z, r)\right) \subset \Omega$, so that $\Delta_{\mathbb{D}}(z, r) \subset$ $\pi^{-1}(\Omega)$. As $z \in \Sigma, \Sigma$ is a component of $\pi^{-1}(\Omega)$, and $\Delta_{\mathbb{D}}(z, r)$ is connected, we conclude that $\Delta_{\mathbb{D}}(z, r) \subset \Sigma$. This gives the reverse inequality to (4.3), and consequently

$$
R(z ; \Sigma, \mathbb{D})=R(\pi(z) ; \Omega, D) .
$$

The conclusion of Lemma 4.2 now follows directly from (4.1).

\section{The proof of Theorem 1.5}

We recall that a subdomain $\Omega$ of $\mathbb{C}$ is a Bloch domain if and only if there is a finite upper bound, say $R_{e}(\Omega, \mathbb{C})$, on the radii of the Euclidean discs lying in $\Omega$. If the supremum of the quotient of the Euclidean metric $|d z|$ on the larger domain $\mathbb{C}$ by the hyperbolic metric on the smaller domain $\Omega$ is $\mu_{e}(\Omega, \mathbb{C})$, that is

$$
\mu_{e}(\Omega, \mathbb{C})=\sup _{z \in \Omega} \frac{|d z|}{\lambda_{\Omega}(z)|d z|},
$$

then

$$
\frac{1}{2} R_{e}(\Omega, \mathbb{C}) \leq \mu_{e}(\Omega, \mathbb{C}) \leq R_{e}(\Omega, \mathbb{C}),
$$


(see [23]) so that $\Omega$ is a Bloch domain if and only if $\mu_{e}(\Omega, \mathbb{C})<\infty$. Theorem 1.5 gives the hyperbolic analogue of these inequalities (for related ideas, see [20, 21]). We break the proof of Theorem 1.5 into four lemmas, the first of which gives the left-hand inequality in Theorem 1.5.

Lemma 5.1. Suppose that $\Omega$ is a subdomain of a hyperbolic domain $D$. Then, for $z$ in $\Omega$,

$$
\tanh \frac{1}{2} R(z ; \Omega, D) \leq \frac{\lambda_{D}(z)}{\lambda_{\Omega}(z)} \leq \mu(\Omega, D) .
$$

Proof. By Lemmas 4.1 and 4.2, we may assume that $D=\mathbb{D}$ and $\Omega \subset \mathbb{D}$. Take any $z_{0}$ in $\Omega$; let $g$ be an automorphism of $\mathbb{D}$ with $g\left(z_{0}\right)=0$ and let $g(\Omega)=\Omega^{\prime}$. Then from Lemmas 4.1 and 4.2 again, $\mu(\Omega, \mathbb{D})=\mu\left(\Omega^{\prime}, \mathbb{D}\right)$ and $R(\Omega, \mathbb{D})=R\left(\Omega^{\prime}, \mathbb{D}\right)$. Suppose now that $\Delta_{\mathbb{D}}(0, t) \subset \Omega^{\prime}$. Then

$$
\frac{\lambda_{\mathbb{D}}(0)}{\lambda_{\Omega^{\prime}}(0)}=\frac{2}{\lambda_{\Omega^{\prime}}(0)} \geq r
$$

where $r$ is the Euclidean radius of $\Delta_{\mathbb{D}}(0, t)$. Thus $r=\tanh \frac{1}{2} t$, and (after taking the supremum over all admissible $t$ ) we conclude that

$$
\frac{\lambda_{\mathbb{D}}(0)}{\lambda_{\Omega^{\prime}}(0)} \geq \tanh \frac{1}{2} R\left(0 ; \Omega^{\prime}, \mathbb{D}\right) .
$$

The conformal invariance (under $g^{-1}$ ) now gives

$$
\frac{\lambda_{\mathbb{D}}\left(z_{0}\right)}{\lambda_{\Omega}\left(z_{0}\right)} \geq \tanh \frac{1}{2} R\left(z_{0} ; \Omega, \mathbb{D}\right),
$$

and this is the first inequality in Lemma 5.1. The second inequality follows immediately from the definition of $\mu(\Omega, D)$.

In order to prove the second inequality in Theorem 1.5 we need two preliminary results. The first of these is an explicit example of a hyperbolic metric, and in this it is convenient to focus on the pseudohyperbolic distance between $z$ and $w$ in $\mathbb{D}$, namely

$$
p(z, w)=\left|\frac{z-w}{1-\bar{w} z}\right|=\tanh \frac{1}{2} \rho_{\mathbb{D}}(z, w) .
$$

Lemma 5.2. Suppose that $c \in \mathbb{D}$ and let $\Delta_{\mathbb{D}}^{*}(c, R)=\Delta_{\mathbb{D}}(c, R) \backslash\{c\}$ (this is the hyperbolic disc in $\mathbb{D}$ with centre $c$ and radius $R$ that is punctured at $c)$. Then

$$
\lambda_{\Delta_{\mathbb{D}}^{*}(c, R)}(z)=\frac{1-p(z, c)^{2}}{2 p(z, c) \log (r / p(z, c))} \lambda_{\mathbb{D}}(z),
$$

where $r=\tanh \frac{1}{2} R$.

Proof. For brevity, we write $\Delta^{*}(c, R)$ instead of $\Delta_{\mathbb{D}}^{*}(c, R)$. The function $T(z)=(z-c) /$ $(1-\bar{c} z)$ is a conformal automorphism of $\mathbb{D}$ that maps $\Delta^{*}(c, R)$ onto $\Delta^{*}(0, R)$. As

$$
\Delta^{*}(0, R)=\{z: 0<|z|<r\}, \quad r=\tanh \frac{1}{2} R,
$$


we see that $g$ defined by $g(z)=r^{-1} T(z)$ maps $\Delta^{*}(c, R)$ conformally onto $\mathbb{D} \backslash\{0\}$. Thus (see [4, p. 17])

$$
\begin{aligned}
\lambda_{\Delta^{*}(c, R)}(z) & =\lambda_{\mathbb{D} \backslash\{0\}}(g(z))\left|g^{\prime}(z)\right| \\
& =\frac{\left|g^{\prime}(z)\right|}{|g(z)| \log (1 /|g(z)|)} \\
& =\frac{\left|T^{\prime}(z)\right|}{|T(z)| \log (r /|T(z)|)},
\end{aligned}
$$

and the result follows as $p(z, c)=|T(z)|$ and $\left|T^{\prime}(z)\right|=\frac{1}{2}\left(1-|T(z)|^{2}\right) \lambda_{\mathbb{D}}(z)$.

The second result that we need is motivated by the function in (5.1), and its proof (which we omit) is a simple exercise in calculus.

LEMMA 5.3. The function

$$
A(k)=k \exp \left(\frac{1-k^{2}}{1+k^{2}}\right)
$$

is increasing on $(0,1)$ and satisfies $A(k)>k$ there. For $0<t<A(k)$, let

$$
h(t)=\frac{1-t^{2}}{2 t \log (A(k) / t)} .
$$

Then $h$ is decreasing on $(0, k]$, increasing on $[k, A(k))$, and satisfies $h(k)=\left(1+k^{2}\right) / 2 k$. In particular, if $k=\tanh y$, then $h(\tanh y)=1 / \tanh (2 y)$.

We now give our final lemma, and this will enable us to complete the proof of Theorem 1.5.

LEMMA 5.4. Suppose that $\Omega$ is a Lipschitz subdomain of a hyperbolic domain $D$ and that $z \in \Omega$. For brevity, write $\tau(z)=\tanh \frac{1}{2} R(z ; \Omega, \mathbb{D})$. Then

$$
\frac{1-\tau(z)^{2}}{2 \tau(z) \log \left(A_{0} / \tau(z)\right)} \lambda_{D}(z) \leq \lambda_{\Omega}(z)
$$

where

$$
A_{0}=\left(\tanh \frac{1}{2} R(\Omega, D)\right) \exp \frac{1-\tanh ^{2} \frac{1}{2} R(\Omega, D)}{1+\tanh ^{2} \frac{1}{2} R(\Omega, D)}=A\left(\tanh \frac{1}{2} R(\Omega, D)\right) .
$$

Consequently, $\mu(\Omega, D) \leq \tanh R(\Omega, D)$.

Proof. As $\Omega$ is a Lipschitz subdomain, $\mu(\Omega, D)<1$ and so, from Lemma 5.1, $R(\Omega, D)<$ $+\infty$. As before, we may assume that $D=\mathbb{D}$ and that $\Omega \subset \mathbb{D}$. Our proof uses Ahlfors' lemma concerning ultrahyperbolic metrics (see $[\mathbf{4}$, p. 13] and [22]), and the example in Lemma 5.2 is used to construct a supporting metric.

We choose $k$ so that

$$
0<\tanh \frac{1}{2} R(\Omega, D)<k<1,
$$

and then (recall that $D=\mathbb{D}$ ) define

$$
\sigma(z)=h(\tau(z)) \lambda_{\mathbb{D}}(z)=\frac{1-\tau(z)^{2}}{2 \tau(z) \log (A(k) / \tau(z))} \lambda_{D}(z) .
$$


The idea of the proof is to show that $\sigma(z)|d z|$ is an ultrahyperbolic metric on $\Omega$. Ahlfors' lemma then implies that $\sigma \leq \lambda_{\Omega}$, and as this inequality is preserved as we let $k$ decrease to $\tanh \frac{1}{2} R(\Omega, D)$, this gives (5.2). Assuming this for the moment, we then have

$$
\begin{aligned}
\frac{\lambda_{D}(z)}{\lambda_{\Omega}(z)} & \leq \frac{2 \tau(z) \log \left(A_{0} / \tau(z)\right)}{1-\tau(z)^{2}} \\
& =\frac{1}{h(\tau(z))} \\
& \leq \frac{1}{h\left(\tanh \frac{1}{2} R(\Omega, D)\right)} \\
& =\tanh R(\Omega, D)
\end{aligned}
$$

which yields the final inequality in Lemma 5.4 (which coincides with the second inequality in Theorem 1.5). It only remains to prove that $\sigma(z)|d z|$ is ultrahyperbolic on $\Omega$.

Select a point $a$ in $\Omega$. We need to demonstrate that $\sigma(z)|d z|$ defined in (5.3) has a supporting metric in a neighborhood of $a$. Choose $c$ in $\mathbb{D} \cap \partial \Omega$ with $\rho_{\mathbb{D}}(a, c)=R(a ; \Omega, \mathbb{D})$. Then for $z$ in $\Omega$ with

$$
\rho_{\mathbb{D}}(z, a)<2 \tanh ^{-1} k-\rho_{\mathbb{D}}(a, c)
$$

(the upper bound here is positive) we have

$$
R(z ; \Omega, \mathbb{D}) \leq \rho_{\mathbb{D}}(z, c) \leq \rho_{\mathbb{D}}(z, a)+\rho_{\mathbb{D}}(a, c)<2 \tanh ^{-1} k,
$$

or equivalently,

$$
\tau(z) \leq \tanh \frac{1}{2} \rho_{\mathbb{D}}(z, c)<k,
$$

with equality when $z=a$. This implies that

$$
\begin{aligned}
\sigma(z) & =h(\tau(z)) \lambda_{\mathbb{D}}(z) \\
& \geq h\left(\tanh \frac{1}{2} \rho_{\mathbb{D}}(z, c)\right) \lambda_{\mathbb{D}}(z) \\
& =h(p(z, c)) \lambda_{\mathbb{D}}(z) \\
& =\lambda_{\Delta^{*}(c, A(k))}(z),
\end{aligned}
$$

with equality at $a$. Thus $\lambda_{\Delta^{*}(c, A(k))}(z)|d z|$ is a supporting metric for $\sigma(z)|d z|$ at $a$ and our proof of Theorem 1.5 is finally complete.

\section{Several complex variables}

We begin by showing that Theorem 1.9 does contain Theorem $\mathrm{C}$, and for this it is sufficient to prove the following lemma.

LEMMA 6.1. Let D be a bounded domain in a complex Banach space $X$, and suppose that $\Omega$ is a relatively compact subdomain of $D$. Then $\Omega$ is a Lipschitz subdomain of $D$.

Proof. Let

$$
\varepsilon=\frac{\operatorname{dist}(\Omega, \partial D)}{2 \operatorname{diam}(\Omega)},
$$

where these terms are computed in the norm $\|\cdot\|$ on $X$. Now take any $(p, v) \in T(\Omega)$ with $v \neq 0$, and let $f: \mathbb{D} \rightarrow \Omega$ be any holomorphic map such that $f(0)=p$ and $d f(0)(x)=v$, 
where $x>0$. Next, define $g: \mathbb{D} \rightarrow X$ by $g(w)=f(w)+\varepsilon(f(w)-p)$. As $f(w) \in \Omega$, and

$$
\|g(w)-f(w)\| \leq \varepsilon \operatorname{diam}(\Omega)<\operatorname{dist}(\Omega, \partial D),
$$

we see that $g$ maps $\mathbb{D}$ into $D$; thus $g \in \mathcal{H}(\mathbb{D}, D)$. Clearly $g(0)=p$, and if $\lambda=1 /(1+\varepsilon)$, then

$$
d g(0)(\lambda x)=(1+\varepsilon) d f(0)(\lambda x)=d f(0)(x)=v,
$$

so we can deduce that

$$
K_{D}(p, v) \leq \lambda x=x /(1+\varepsilon) .
$$

As $f$ is arbitrary, and $\varepsilon$ is independent of $f$, this implies that

$$
K_{D}(p, v) \leq \frac{1}{1+\varepsilon} K_{\Omega}(p, v) .
$$

As $(p, v)$ is any point in $T(\Omega)$ and $v \neq 0$, we deduce that $\mu(\Omega, D) \leq 1 /(1+\varepsilon)<1$ as required.

Next, we give the proof of Theorem 1.9, and this follows much as before except that the metric space $\left(D, k_{D}\right)$ need not be complete (see [12, p. 81]). Note that in the case of one complex variable, $k_{D}=\rho_{D}$ and completeness is assured. This difficulty is overcome by following the ideas in the proof of Theorem B in [14] which is based on [14, Lemma V.5.1, p. 137].

Proof of Theorem 1.9. First, the obvious analogue of Lemma 3.1 holds in the circumstances given here, but we cannot use Theorem 1.1 directly as the space $\left(D, k_{D}\right)$ might not be complete. However, the argument used to prove Theorem 1.1 does show that, for any $x$ in $D$, the sequence $f_{1} \circ \cdots \circ f_{n}(x)$ is a Cauchy sequence with respect to $k_{D}$. As $D$ is bounded then there is some positive $R$ such that for any $x$ in $D, D$ lies in the open ball $B(x, R)$, and using this and the monotonicity of the metric as a function of the domain, we find that if $x$ and $y$ are in $D$, then

$$
k_{D}(x, y) \geq k_{B(x, R)}(x, y)=2 \tanh ^{-1}\left(\frac{\|x-y\|}{R}\right) .
$$

It follows that for any $x$ in $D$, the sequence $f_{1} \circ \cdots \circ f_{n}(x)$ is a Cauchy sequence with respect to the norm of the underlying Banach space $X$ and so converges in $X$ to some value, say $g(x)$. Now take any $x$ and $y$ in a compact subset of $D$ and observe that

$$
k_{D}\left(f_{1} \circ \cdots \circ f_{n}(x), f_{1} \circ \cdots \circ f_{n}(y)\right) \leq \mu(\Omega, D)^{n} k_{D}(x, y) \rightarrow 0
$$

as $n \rightarrow \infty$. Thus $g(x)=g(y)$, and as this implies that $g$ is constant on $X$, the proof is complete.

We remark that the proof of Theorem 1.9 shows that if $f_{1}, f_{2}, \ldots$ are holomorphic maps from a bounded subdomain $D$ of $X$ into a Lipschitz subdomain $\Omega$ of $D$, then for each $x$ in $D$, the sequence $f_{1} \circ \cdots \circ f_{n}(x)$ either converges to a point $\zeta$ in $D$, or it accumulates only on $\partial D$. Moreover, the two possibilities here are independent of the choice of $x$ and, in the former case, $\zeta$ is also independent of $x$. 
7. An example

We end this paper with an example of a domain $\Omega$ in $\mathbb{C}^{2}$ that is a Bloch domain but not a Lipschitz domain (both with respect to the Kobayashi metric). As our example is in $\mathbb{C}^{2}$, it is convenient to begin with some general remarks about Lipschitz subdomains of product spaces.

First, we note that if $U$ and $V$ are subdomains of the complex Banach spaces $X$ and $Y$, respectively, then [14, Proposition V.1.6, p. 117]

$$
K_{U \times V}((z, w),(u, v))=\max \left\{K_{U}(z, u), K_{V}(w, v)\right\} .
$$

Suppose now that $\Omega_{j}$ is a Lipschitz subdomain of $D_{j}, j=1,2$, then

$$
\frac{K_{D_{1} \times D_{2}}\left(\left(p_{1}, p_{2}\right),\left(v_{1}, v_{2}\right)\right)}{K_{\Omega_{1} \times \Omega_{2}}\left(\left(p_{1}, p_{2}\right),\left(v_{1}, v_{2}\right)\right)}=\frac{\max \left\{K_{D_{1}}\left(p_{1}, v_{1}\right), K_{D_{2}}\left(p_{2}, v_{2}\right)\right\}}{\max \left\{K_{\Omega_{1}}\left(p_{1}, v_{1}\right), K_{\Omega_{2}}\left(p_{2}, v_{2}\right)\right\}} \text {. }
$$

Now for any positive numbers $a_{i}$ and $b_{j}$,

$$
\frac{\max \left\{a_{1}, a_{2}\right\}}{\max \left\{b_{1}, b_{2}\right\}} \leq \max \left\{\frac{a_{1}}{b_{1}}, \frac{a_{2}}{b_{2}}\right\},
$$

so the next result follows immediately.

THEOREM 7.1. Suppose that $\Omega_{j}$ is a Lipschitz subdomain of $D_{j}, j=1,2$, and that each $D_{j}$ is Kobayashi hyperbolic. Then $\Omega_{1} \times \Omega_{2}$ is a Lipschitz subdomain of $D_{1} \times D_{2}$.

This lemma enables us to construct many Lipschitz subdomains of, for example, $\mathbb{D}^{N}$, and hence to provide many examples to which Theorem 1.9 is applicable.

Finally, given $X$ and $Y$, let $\pi_{j}$ be the projections given by $\pi_{1}(z, w)=z$ and $\pi_{2}(z, w)$ $=w$. Then we have the following result.

THEOREM 7.2. Suppose that $\Omega \subset D_{1} \times D_{2}$, where each $D_{j}$ is Kobayashi hyperbolic. If $\pi_{j}(\Omega)$ is a Lipschitz subdomain of $D_{j}, j=1,2$, then $\Omega$ is a Lipschitz subdomain of $D_{1} \times D_{2}$.

Proof. First, $\Omega \subset \pi_{1}(\Omega) \times \pi_{2}(\Omega)$. By Theorem 7.1 this product set is Lipschitz, and hence so is any subdomain of it.

We now construct our example. First, we construct a sequence $z_{n}$ in the unit disc $\mathbb{D}$ in $\mathbb{C}$ such that $\left|z_{n}\right| \rightarrow 1$ and such that if $E=\left\{z_{1}, z_{2}, \ldots\right\}$, then every point of $\mathbb{D}$ is within a distance of one of $E$ when measured in the hyperbolic metric $\rho_{\mathbb{D}}$ of $\mathbb{D}$. We show that $\Omega$ defined by

$$
\Omega=(\mathbb{D} \times \mathbb{D}) \backslash(E \times E)
$$

is a Bloch, but not a Lipschitz, domain. First, $\Omega$ is a Bloch domain in $\mathbb{D} \times \mathbb{D}$ because

$$
\begin{aligned}
k_{\mathbb{D} \times \mathbb{D}}\left((z, w),\left(z_{p}, z_{q}\right)\right) & =\max \left\{k_{\mathbb{D}}\left(z, z_{p}\right), k_{\mathbb{D}}\left(w, z_{q}\right)\right\} \\
& =\max \left\{\rho_{\mathbb{D}}\left(z, z_{p}\right), \rho_{\mathbb{D}}\left(w, z_{q}\right)\right\} \\
& \leq 1
\end{aligned}
$$

for a suitable choice of $p$ and $q$ (see [14, p. 136]). 
To show that $\Omega$ is not Lipschitz we use a result of Kobayashi [17, Proposition 3.5.35, p. 96], which states that if $A$ is a closed analytic subset of codimension two in a complex manifold $M$, then

$$
K_{M \backslash A}(p, v)=K_{M}(p, v)
$$

when $p \in M \backslash A$. In particular, $\mu(M \backslash A, M)=1$, so that $M \backslash A$ is certainly not Lipschitz. We take $M=\mathbb{D} \times \mathbb{D}$ and $A=E \times E$, and as $E \times E$ is obviously closed and of codimension two in $\mathbb{D} \times \mathbb{D}$, it is sufficient to show that it is an analytic subset of $\mathbb{D} \times \mathbb{D}$. The assumptions on the $z_{n}$ in $\mathbb{D}$ guarantee that there is an analytic function $f: \mathbb{D} \rightarrow \mathbb{C}$ that is zero on, and only on, $E\left[14\right.$, p. 295], so we may define functions $f_{1}(z, w)=f(z)$ and $f_{2}(z, w)=f(w)$ that are analytic on $\mathbb{D} \times \mathbb{D}$. As

$$
E \times E=f_{1}^{-1}(\{0\}) \cap f_{2}^{-1}(\{0\}),
$$

we see that $E \times E$ is indeed an analytic subset of $\mathbb{D} \times \mathbb{D}$.

Acknowledgement. T. W. Ng was partially supported by RGC Grant HKU 7020/03P.

\section{REFERENCES}

[1] M. Abate. Iteration Theory of Holomorphic Maps on Taut Manifolds. Mediterranean Press, Rende, 1989.

[2] A. Ambroladze. Ergodic properties of random iterations of analytic functions. Ergod. Th. \& Dynam. Sys. 19 (1999), 1379-1388.

[3] A. Ambroladze and H. Wallin. Random iteration of isometries of the hyperbolic plane. Markov Process. Related Fields 5 (1999), 69-88.

[4] L. V. Ahlfors. Conformal Invariants. McGraw-Hill, New York, 1973.

[5] I. N. Baker and P. J. Rippon. Towers of exponents and other composite maps. Complex Variables 12 (1989), 181-200.

[6] I. N. Baker and P. J. Rippon. On compositions of analytic self-mappings of a convex domain. Arch. Math. 55 (1990), 380-386.

[7] A. F. Beardon. Iteration of contractions and analytic maps. J. London Math. Soc. 41(2) (1990), 141-150.

[8] A. F. Beardon. Iteration of analytic Euclidean contractions. Computational Methods and Function Theory. Eds R. M. Ali, St. Ruscheweyh and E. B. Saff. World Scientific, Singapore, 1995, pp. 57-74.

[9] A. F. Beardon. Analytic contractions of the unit disc. Bull. Hong Kong Math. Soc. 1 (1997), 209-218.

[10] A. F. Beardon. The dynamics of contractions. Ergod. Th. \& Dynam. Sys. 17 (1997), 1257-1266.

[11] R. Brück, M. Büger and S. Reitz. Random iterations of polynomials of the form $z^{2}+c_{n}$; connectedness of Julia sets. Ergod. Th. \& Dynam. Sys. 19 (1999), 1221-1231.

[12] S. Dineen. The Schwarz Lemma. Oxford University Press, Oxford, 1989.

[13] C. J. Earle and R. S. Hamilton. A fixed point theorem for holomorphic mappings. Proc. Symp. Pure Math. 16 (1979), 61-65.

[14] T. Franzoni and E. Vesentini. Holomorphic Maps and Invariant Distances (North-Holland Mathematical Studies, 40). North-Holland, Amsterdam, 1980.

[15] J. Gill. Compositions of analytic functions of the form $F_{n}(z)=F_{n-1}\left(f_{n}(z)\right), f_{n}(z) \rightarrow f(z)$. J. Comput. Appl. Math. 23(2) (1988), 179-184.

[16] J. Gill. Inner composition of analytic mappings on the unit disk. Int. J. Math. Math. Sci. 14 (1991), 221226.

[17] S. Kobayashi. Hyperbolic Complex Spaces. Springer, Berlin, 1998.

[18] L. Lorentzen. Compositions of contractions. J. Comput. Appl. Math. 32 (1990), 169-178.

[19] L. Lorentzen. Convergence of compositions of self-mappings. Ann. Univ. Marie Curie Sklodowska A 53(13) (1999), 121-145. 
[20] D. Minda. Bloch constants. J. d'Analyse Math. 41 (1982), 54-84.

[21] D. Minda. Estimates for the hyperbolic metric. Kodai Math. J. 8 (1985), 249-258.

[22] D. Minda. A reflection principle for the hyperbolic metric and applications to geometric function theory. Complex Variables 8 (1987), 129-144.

[23] Ch. Pommerenke. On Bloch functions. J. London Math. Soc. 2(2) (1970), 689-695.

[24] H. Royden. Remarks on the Kobayashi metric. Proc. Maryland Conference on Several Complex Variables (Lecture Notes in Mathematics, 185). Springer, Berlin, 1971, pp. 369-383.

[25] W. J. Zhang and F. Y. Ren. Random iteration of holomorphic self-maps over bounded domains in $\mathbb{C}^{n}$. Chinese Ann. Math. 16B (1995), 33-42. 